\title{
Eigenvalue Spectrum and Synchronizability of Double-Layer Directed Ring Networks
}

\author{
Ping Wu, Zhen Jia*, Feimei Yang, Jiaquan Huang \\ College of Science, Guilin University of Technology, Guilin, China \\ Email: ‘jjjzzz0@163.com
}

How to cite this paper: $\mathrm{Wu}, \mathrm{P}$., Jia, Z., Yang, F.M. and Huang, J.Q. (2021) Eigenvalue Spectrum and Synchronizability of Double-Layer Directed Ring Networks. Journal of Applied Mathematics and Physics, 9, 3067-3087.

https://doi.org/10.4236/jamp.2021.912199

Received: November 8, 2021

Accepted: December 18, 2021

Published: December 21, 2021

Copyright $\odot 2021$ by author(s) and Scientific Research Publishing Inc. This work is licensed under the Creative Commons Attribution International License (CC BY 4.0).

http://creativecommons.org/licenses/by/4.0/

\begin{abstract}
The synchronizability of multiplex undirected regular networks has been intensively studied based on the study of the synchronizability of single-layer networks. However, most real networks are characterized by some degree of directionality. So multiplex directed networks can better explain the synchronizability phenomenon. Here, based on the theory of master stability function (MSF), we study the eigenvalue spectrum and synchronizability of double-layer inter-layer directed ring networks (Networks-A) and double-layer intra-layer directed ring networks (Networks-B). The eigenvalue spectrum of the supra-Laplacian matrix of the networks is rigorously derived, and the influence of the networks structure parameters on the network's synchronizability is analyzed. The correctness of the theory is further verified by numerical simulation analysis. Finally, the synchronizability of four kinds of double-layer ring networks with different coupling modes, namely, Networks-A, Networks-B, Networks-C (double-layer undirected ring networks), and Networks-D (double-layer undirected inter-layer random-added-edge ring networks), is compared and the results can provide guidance for constructing the optimal synchronization network.
\end{abstract}

\section{Keywords}

Double-Layer Ring Networks, Eigenvalue Spectrum, Synchronizability

\section{Introduction}

In the last two decades, research on complex networks has developed rapidly and many important results have been achieved. Actual complex systems are composed of multiple networks coupled and interacting with each other [1] [2] [3], for example, interpersonal social networks, neural networks, ecological networks composed of species interactions, etc. [4] [5] [6]. The multiplex networks 
model has been proposed internationally in recent years and has become one of the most important research directions at the forefront of the current complex network landscape [7]. The interaction between different complex systems leads to the emergence of multiplex complex networks. Synchronization is a very common phenomenon in nature. Previous research has been conducted on different synchronization effects, such as full synchronization, intra-layer synchronization, inter-layer synchronization, etc. [8]-[14].

Synchronizability plays an overwhelming role in research fields such as biology, chemistry, physics, and economics. Synchronizability of multi-layer networks is of great theoretical and economic importance, while the study is still in its infancy [15]-[22]. Among them, the Synchronizability of multiplex-directed networks has been less researched. A ring network is a type of regular network. Many technical networks, such as sensor networks and robot networks have the structural characteristics of ring networks, so it is of practical and economic importance to explore the synchronization of ring networks [23]. In terms of research on the synchronization of ring networks, in 2017, Wei Juan et al. studied the potential factors affecting the synchronization of two-layer regular networks, including the scale of the networks, inter-layer coupling strength, and intra-layer coupling strength, based on the master stability function (MSF) method and numerical simulation analysis. Among them, there are two modes of inter-layer connections, one is a one-to-one correspondence between layers with full connections and the other one is between layers with one corresponding edge connected, it was found that for a ring network with fixed coupling strength, the more the number of inter-layer connections, the better the synchronization [24]. In 2017, Sun Juan et al. derived the eigenvalue spectrum of a multilayer unidirectional star network based on the MSF and analyzed the factors affecting its network synchronization ability [25]. In 2019, Yang Lixin et al. studied the effect of the interconnection mode of a multiplex oscillator grid consisting of two subnetworks on the synchronization ability, which found that different interconnection modes affect the synchronization ability of the whole network. It revealed that increasing the number of inter-layer connections between ring networks of the same property facilitates the synchronization of double-layer networks [26]. In 2020, Zhang Li et al. derive an analytic expression for the eigenvalue spectrum of a multilayer k-nearest neighbor coupled network with one-to-one corresponding full connectivity between layers in terms of the master stability function and analyzed the effect of the network structure parameters on the synchronization ability of this network [27]. In 2021, Yang Feimei et al. rigorously derived the eigenvalue spectrum of two types of double-layers directed star-ring networks and analyzed the parameters that affect the synchronizability of the networks [28]. In summary, we find that the synchronizability of the ring networks is mostly studied by numerical simulation and comparison with other regular networks, and there is still a space for a rigorous theoretical derivation of the synchronization capability of multilayer directed ring networks. Studies on the synchronizability of multilayer networks have found that the inter-layer connec- 
tion density, inter-layer coupling weight, intra-layer coupling weight, and the scale of the network impact the synchronization ability of the networks. In the actual network, the direction and coupling weight is more sophisticated, following which we consider how the degree of influence of the directionality of the networks and the number of inter-layer connections on the synchronizability of the networks?

In this paper, we rigorously and strictly derive the eigenvalue spectrum of Networks-A and Networks-B based on the theory of MSF and analyze the effects of intra-layer coupling weight $a$, inter-layer coupling weight $d$, and the number of nodes within each layer $N$ on the synchronizability. Finally, simulations are undertaken to analyze the network synchronizability of four kinds of Networks-A, Networks-B, Networks-C, and Networks-D. It is organized as follows: the multilayer network dynamics model and the network structure model are given in Section 2. The eigenvalue spectra of Networks-A and Networks-B and their eigenvalue analytical expressions are rigorously derived in Sections 3 and 4 , and numerical simulations are performed to verify the correctness of the theoretical results. Section 5 analyzes and compares the synchronizability of four kinds of double-layer ring networks with numerical simulations. Conclusions are given in the last section.

\section{Preliminaries}

\subsection{The Dynamics Model of Multilayer Network}

For a multiplex network consisting of $M$ layers and $N$ nodes each layer, the dynamics of $x_{i}^{\alpha}$ can be described as [29] [30]:

$$
\frac{\mathrm{d} x_{i}^{\alpha}}{\mathrm{d} t}=f_{i}^{\alpha}\left(x_{i}^{\alpha}\right)+\sum_{j=1}^{N} a_{i j}^{\alpha} \Gamma_{1}\left(x_{j}^{\alpha}-x_{i}^{\alpha}\right)+\sum_{\beta=1}^{M} d_{i}^{\alpha \beta} \Gamma_{2}\left(x_{i}^{\beta}-x_{i}^{\alpha}\right),
$$

where $1 \leq i \leq N, \quad 1 \leq \alpha \leq M, \quad x_{i}^{\alpha} \in \mathfrak{R}^{n}$ is the state vector of the $i$ th node in the $\alpha$ th layer. $f_{i}^{\alpha}: \mathfrak{R}^{n} \rightarrow \mathfrak{R}^{n}$ is the dynamic equation of the $i$ th node in the $\alpha$ th layer, $\Gamma_{1}: \Re^{n} \rightarrow \mathfrak{R}^{n}$ is the intra-layer coupling function defining the interaction between nodes in the same layer, and $\Gamma_{2}: \mathfrak{R}^{n} \rightarrow \mathfrak{R}^{n}$ is the inter-layer coupling function defining the interaction between nodes on a separate layer. For simplicity, let $f_{i}^{\alpha}=f$ and $\Gamma_{1}=\Gamma_{2}=\Gamma$. The intra-layer coupling weight $a_{i j}^{\alpha}$ is positive if the $i$ th node is connected with the $j$ th node in the $\alpha$ th layer $(i \neq j)$, otherwise $a_{i j}^{\alpha}=0$, and there is

$$
a_{i i}^{\alpha}=\sum_{j=1, i \neq j}^{N} a_{i j}^{\alpha}, \quad i, j=1,2, \cdots, N ; \alpha=1,2, \cdots, M .
$$

If the $i$ th node in the $\alpha$ th layer is connected to the $j$ node in the $\beta$ th layer $(\alpha \neq \beta)$, then the interlayer coupling weight $d_{i}^{\alpha \beta}$ is negative, otherwise $d_{i}^{\alpha \beta}=0$, and there is

$$
d_{i}^{\alpha \alpha}=-\sum_{\beta=1, \alpha \neq \beta}^{M} d_{i}^{\alpha \beta}, \alpha, \beta=1,2, \cdots, M ; i=1,2, \cdots, N .
$$

Let $\mathcal{L}$ is the supra-Laplacian matrix of multiplex networks, $\mathcal{L}^{L}$ is the in- 
tra-layer supra-Laplacian matrix, $\mathcal{L}^{I}$ is the inter-layer supra-Laplacian matrix, then $\mathcal{L}, \mathcal{L}^{L}, \mathcal{L}^{I}$ can be written as:

$$
\mathcal{L}=\mathcal{L}^{L}+\mathcal{L}^{I}
$$

Among them,

$$
\mathcal{L}^{L}=\left(\begin{array}{cccc}
L^{(1)} & 0 & \cdots & 0 \\
0 & L^{(2)} & \cdots & 0 \\
\vdots & \vdots & \ddots & \vdots \\
0 & 0 & \cdots & L^{(M)}
\end{array}\right)=\bigoplus_{\alpha=1}^{M} L^{(\alpha)} .
$$

$L^{(\alpha)} \in \mathfrak{R}^{N \times N}$ is the intra-layer Laplacian matrix of the $\alpha$ th layer. $\oplus$ is the direct sum operation, $L^{(\alpha)}$ can be written as:

$$
\begin{aligned}
L_{i, j}^{(\alpha)} & =\left\{\begin{array}{l}
-a_{i j}^{(\alpha)}, i \neq j \\
a_{i i}^{(\alpha)}, i=j
\end{array}\right. \\
\mathcal{L}^{I} & =L^{I} \otimes I_{N} .
\end{aligned}
$$

$\otimes$ is the Kronecker product, $L^{I}=\left(d_{i}^{\alpha \beta}\right) \in \mathfrak{R}^{M \times M}$ is the inter-layer Laplacian matrix, $I_{N}$ is the $N \times N$ identity matrix.

Eigenvalues of the supra-Laplacian matrix of the networks are recorded as: $0=\lambda_{1}<\lambda_{2} \leq \cdots \leq \lambda_{\max }$. based on the theory of master stability function (MSF), A network can be achieving synchronization when all eigenvalues of its Laplacian matrix fall within the synchronized region of that network. The synchronization region of a real network can be mainly divided into two kinds: unbounded synchronized region and bounded synchronized region (other cases such as concurrent synchronized region of multiple unconnected intervals and empty region rarely occur, and only two cases of unbounded and bounded synchronized region are studied in this paper). In general, the synchronizability of the network is determined by the minimum non-zero eigenvalue $\lambda_{2}$ or the ratio $R=\lambda_{\max } / \lambda_{2}$ of the maximum eigenvalue to the minimum non-zero eigenvalue of the supra-Laplacian matrix $\mathcal{L}$. When the network synchronization region is unbounded, the larger $\lambda_{2}$ is, the stronger the synchronizability of the network; when the network synchronized region is bounded, the smaller $R=\lambda_{\max } / \lambda_{2}$ is, the stronger the synchronizability of the network.

To convenient the following theoretical derivation, two lemmas are given here:

Lemma 1 ([31]). If $A$ is a square matrix of degree $n, D$ is a square matrix of degree $m, O$ is $m \times n$ zero matrices, and $B$ is a $n \times m$ matrix, then

$$
\left|\begin{array}{ll}
A & B \\
O & D
\end{array}\right|=|A| \cdot|D| .
$$

Lemma 2 ([32]). Let $A, B, C$ and $D$ be all square matrices of the same dimension, and $A C=C A$, then

$$
\left|\begin{array}{ll}
A & B \\
C & D
\end{array}\right|=|A D-C B| .
$$




\subsection{Structural Model of Double-Layer Ring Networks}

In this paper, we focus on the synchronizability of inter-layer directed doublelayer ring networks and intra-layer directed double-layer ring networks. It is supposed that the topology of each layer of the double-layer ring network is identical. The number of nodes within each layer $N$, the inter-layer coupling weight $d$, and the intra-layer coupling weight $a$ is all the same in the networks. Number each layer node orderly and form node pairs with the same number. The layer interconnection method of double-layer inter-layer directed ring networks is a unidirectional connection between layer node pairs as shown in Figure 1(a), double-layer intra-layer directed ring networks means that the nodes of the two layers are connected in opposite sequence as shown in Figure 1(b), double-layer undirected ring networks as shown in Figure 1(c), doublelayer undirected inter-layer random-added-edge ring networks is based on the double-layer undirected ring networks with randomly connected undirected edges of interlayer nodes, the interlayer connected edge probability is $p(0 \leq p \leq 1)$ as shown in Figure $1(d)$.

For clarity of description, we denote the double-layer inter-layer directed ring networks as Networks-A, double-layer intra-layer directed ring networks as Networks-B, double-layer undirected ring networks as Networks-C, and double-layer undirected inter-layer random-added-edge ring networks as Networks-D. Additionally, $S y n_{A}$ denotes the synchronizability of Networks-A, and so forth.

\section{Analysis of the Synchronizability of Networks-A}

\subsection{The Eigenvalue Spectrum and Synchronizability of Networks-A}

From the structural model in Figure 1(a), the supra-Laplacian matrix corresponding to Networks-A can be expressed as:

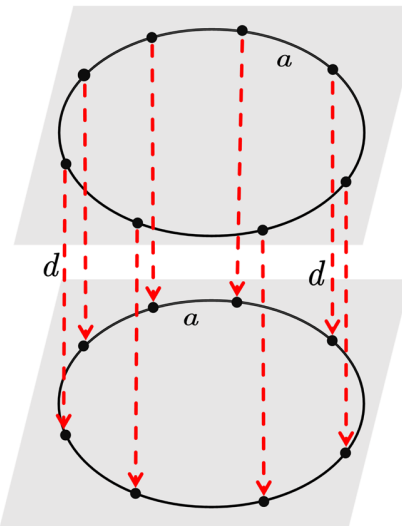

(a)

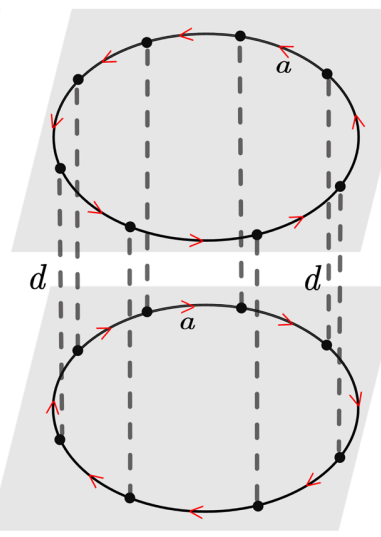

(b)

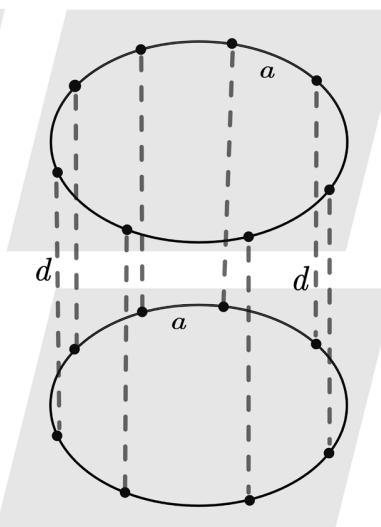

(c)

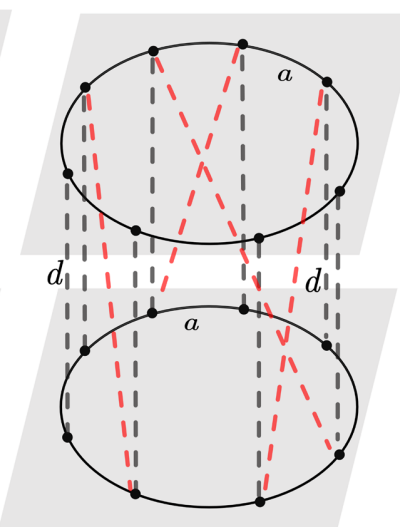

(d)

Figure 1. Schematic diagram of the structure of double-layer ring network. (a) double-layer inter-layer directed ring networks; (b) double-layer intra-layer directed ring networks; (c) double-layer undirected ring networks; (d) double-layer undirected inter-layer random-added-edge ring networks. 


$$
\mathcal{L}_{1}=\left(\begin{array}{cc}
A+d I_{N} & -d I_{N} \\
O & A
\end{array}\right)
$$

where

$$
A=\left(\begin{array}{cccc}
2 a & -a & \overbrace{0 \cdots 0}^{N-3} & -a \\
-a & 2 a & -a & \overbrace{0 \cdots 0}^{N-3} \\
\vdots & \ddots & \ddots & \ddots \\
-a & \overbrace{0 \cdots 0}^{N-3} & -a & 2 a
\end{array}\right) .
$$

According to Lemma 1, the Characteristic polynomials of $\mathcal{L}_{1}$ is:

$$
\left|\lambda I_{2 N}-\mathcal{L}_{1}\right|=\left|\lambda I_{N}-A\right| \cdot\left|(\lambda-d) I_{N}-A\right| .
$$

Let $\left|\lambda I_{N}-A\right|=0,\left|(\lambda-d) I_{N}-A\right|=0$, the eigenvalues of $\mathcal{L}_{1}$ can be written as [27]:

$$
\lambda_{l}=4 a \sin ^{2}\left(\frac{(l-1) \pi}{N}\right), 4 a \sin ^{2}\left(\frac{(l-1) \pi}{N}\right)+d, \quad l=1,2, \cdots, N .
$$

when $N$ is odd, at $l=\frac{N-1}{2}+1$ or $l=\frac{N+1}{2}+1$ has a maximum value of $\lambda$ :

$$
\lambda_{\max }=4 a \cos ^{2} \frac{\pi}{2 N}+d .
$$

when $N$ is even, at $l=\frac{N}{2}+1$ has a maximum value of $\lambda$ :

$$
\lambda_{\text {max }}=4 a+d .
$$

In practice, the number of network sizes is huge and for simplicity we take:

$$
\begin{gathered}
\lambda_{2}=\min \left\{d, \frac{4 a \pi^{2}}{N^{2}}\right\}, \\
\lambda_{\max } \approx 4 a+d, \\
R \approx \frac{4 a+d}{\min \left\{d, \frac{4 a \pi^{2}}{N^{2}}\right\}} .
\end{gathered}
$$

According to the MSF theory, the relationship between the synchronizability of Networks-A and the structural parameters is shown in Table 1.

\subsection{Numerical Simulation of the Synchronizability of Networks-A}

In this paper, the values of various parameters verified by numerical simulation are set within the allowed range.

From Figure 2(a), when the unbounded synchronized region, it is clear that the value of $\lambda_{2}$ increases linearly at first with the increase of $a$ (when $a<d N^{2} / 4 \pi^{2}$ ), and then remains a fixed value $\lambda_{2}=d=0.01$ with the increase of $a$ (when $a>d N^{2} / 4 \pi^{2}$ ). This means that the synchronizability of Networks- $\mathrm{A}$ is first strengthened and then remains constant with the increase in $a$. 
Table 1. The change of synchronizability of Networks-A with the increase of $a, d, N$.

\begin{tabular}{|c|c|c|c|c|c|}
\hline & & & \multicolumn{3}{|c|}{ Increase of } \\
\hline & & & $a$ & $d$ & $N$ \\
\hline \multirow{2}{*}{$\begin{array}{c}\text { The case of the } \\
\text { synchronized region } \\
\text { unbounded }\end{array}$} & $d<\frac{4 a \pi^{2}}{N^{2}}$ & $\lambda_{2}=d$ & - & $\uparrow$ & - \\
\hline & $d>\frac{4 a \pi^{2}}{N^{2}}$ & $\lambda_{2} \approx \frac{4 a \pi^{2}}{N^{2}}$ & $\uparrow$ & - & $\downarrow$ \\
\hline \multirow{2}{*}{$\begin{array}{c}\text { The case of the } \\
\text { synchronized region } \\
\text { bounded }\end{array}$} & $d<\frac{4 a \pi^{2}}{N^{2}}$ & $R \approx \frac{4 a+d}{d}$ & $\downarrow$ & $\uparrow$ & - \\
\hline & $d>\frac{4 a \pi^{2}}{N^{2}}$ & $R \approx \frac{(4 a+d) N^{2}}{4 a \pi^{2}}$ & $\uparrow$ & $\downarrow$ & $\downarrow$ \\
\hline
\end{tabular}

—: unchanged; $\uparrow:$ strengthen; $\downarrow$ : weaken.
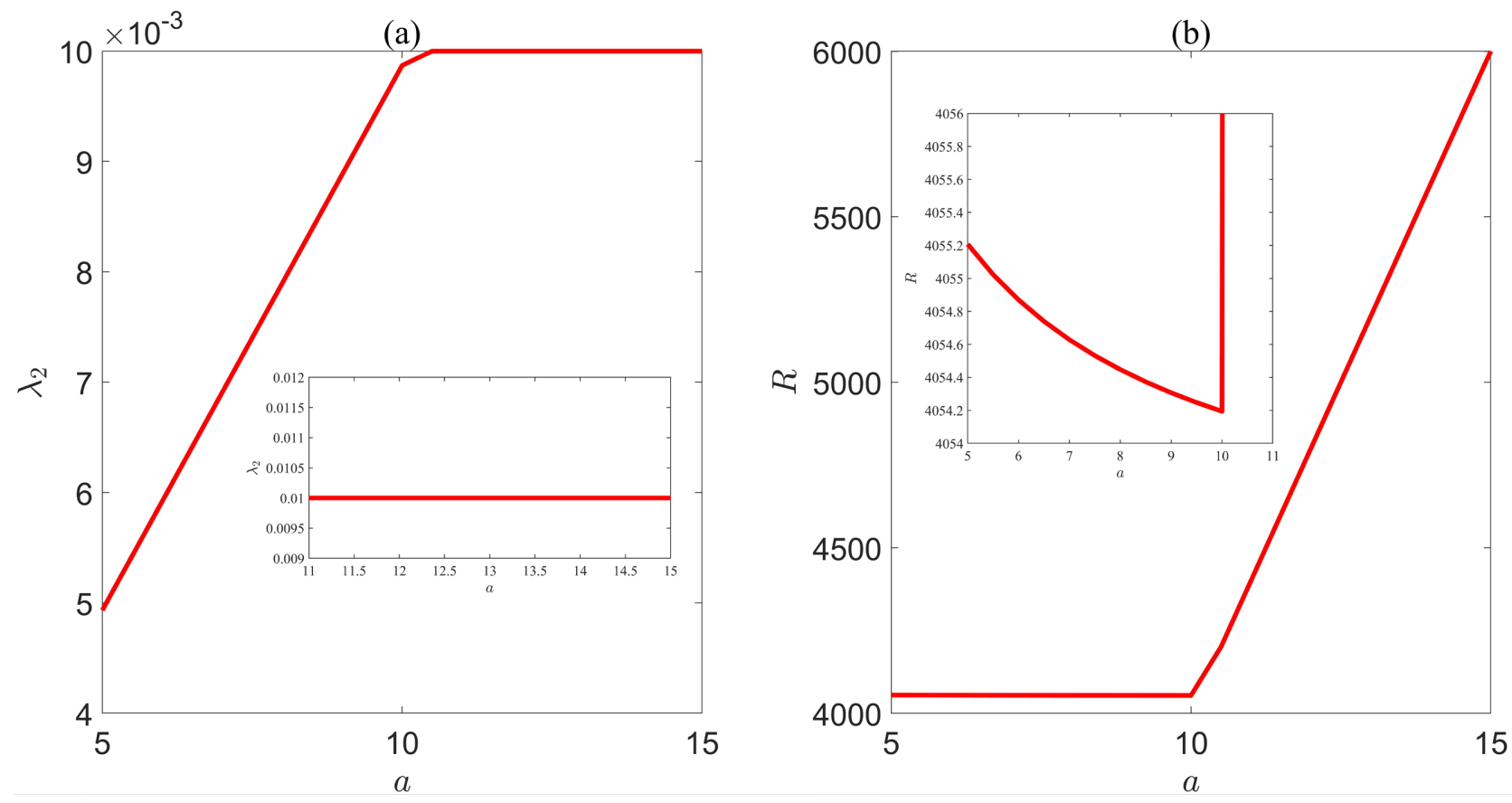

Figure 2. The synchronizability of Networks-A vs. varying intra-layer coupling weight $a(N=200, d=0.01)$. (a) $\lambda_{2}$ vs. varying $a$ ( $a$ varies from 5 to 15) (subgraph: $\lambda_{2}$ vs. varying $a$ ( $a$ varies from 11 to 15)); (b) $R$ vs. varying $a$ ( $a$ varies from 5 to 15) (subgraph: $R$ vs. varying a ( $a$ varies from 5 to 11)).

From Figure 2(b), when the bounded synchronized region, The value of $R$ is first decreases slowly (when $a<d N^{2} / 4 \pi^{2}$ ) and then increases with the increase of $a$ (when $a>d N^{2} / 4 \pi^{2}$ ). This indicates that the synchronizability of the Networks-A is first strengthened and then continuously weakened with an increase in intra-layer coupling weight $a$. The synchronizability of Networks-A is optimum at $a^{*}=d N^{2} / 4 \pi^{2}$.

As is shown in Figure 3, when $d<4 a \pi^{2} / N^{2}$, the value of $\lambda_{2}\left(\lambda_{2}=d\right)$ increases with the increase of $d$ and the value of $R(R=1+(4 a / d))$ decreases with the increase of $d$, with the synchronized region is unbounded. When 

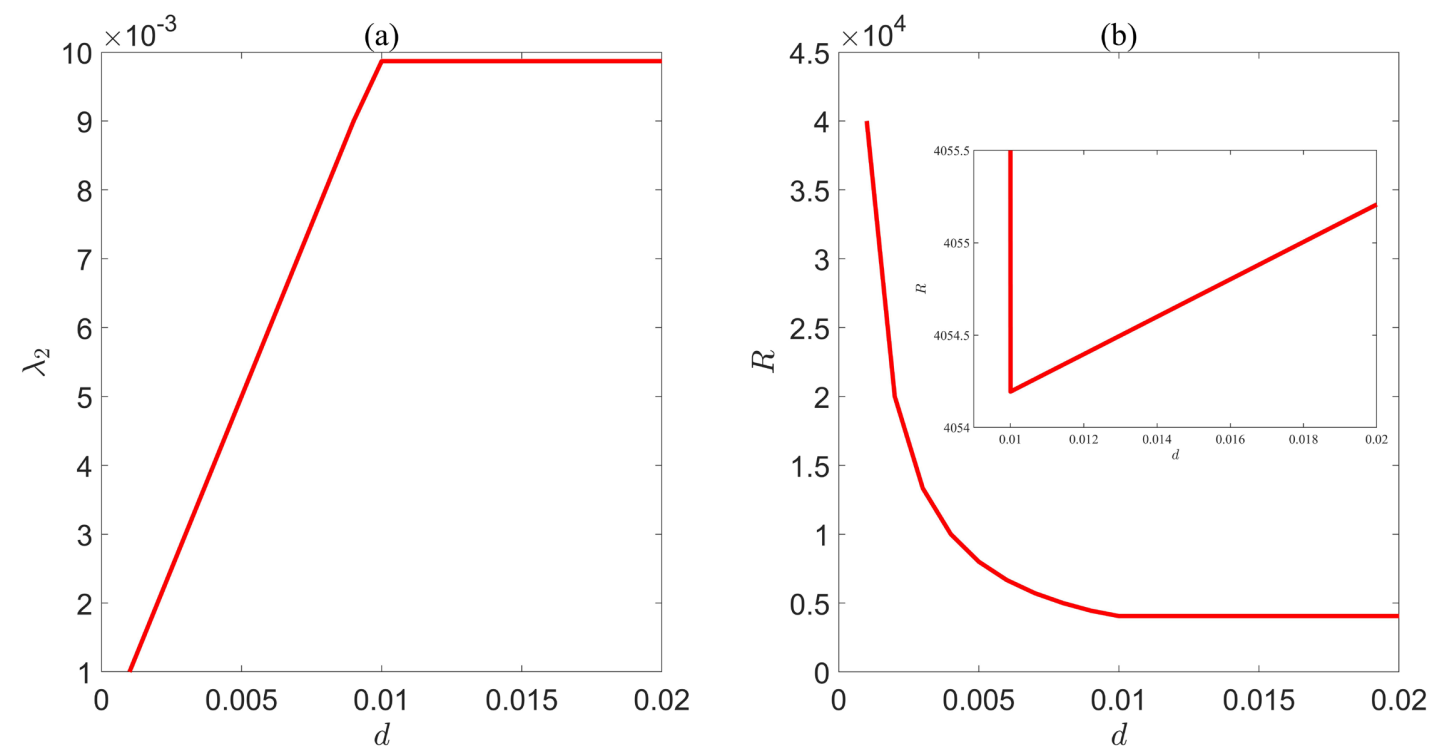

Figure 3. The synchronizability of Networks-A vs. varying inter-layer coupling weight $d(N=200, a=10)$. (a) $\lambda_{2}$ vs. varying $d$ ( $d$ varies from 0.001 to 0.02 ); (b) $R$ vs. varying $d$ ( $d$ varies from 0.001 to 0.02 ) (subgraph: $R$ vs. varying $d$ ( $d$ varies from 0.009 to 0.02$)$ ).

$d>4 a \pi^{2} / N^{2}$, the value of $\lambda_{2}$ does not increase with the increase of $d$, the value of $R$ enhances with the increase of $d$. Hence, with the increase of $d$, the synchronizability of Networks-A is strengthened at first and then remains stable when the synchronized region is unbounded. When the synchronized region is bounded, the synchronizability of Networks-A is first weakened continuously and then strengthened slightly. The synchronizability of Networks-A is optimum at $d^{*}=4 a \pi^{2} / N^{2}$.

Figure 4(a) shows that with the unbounded synchronized region, the value $\lambda_{2}$ remains invariant at first with the increase of $N$ (when $N<\sqrt{4 a \pi^{2} / d}$ ) and then decreases with the increase of $N$ (when $N>\sqrt{4 a \pi^{2} / d}$ ), so the synchronizability of Networks-A first remains unchanged and then weakens. With bounded synchronized region, when $N<\sqrt{4 a \pi^{2} / d}$, as shown in the subgraph of Figure 4(b), when $N$ is even, the value of $R(R=(4 a+d) / d=2001)$ remains unchanged with the increase of $N$, and then when $N$ is odd, the value of $R$ $\left(R=\left[d+4 a \cos ^{2}\left(\pi / 2 N^{2}\right)\right] / d\right)$ increases with the increase of $N$, so the value of $R$ is less and less affected by $N$ being odd and even. Hence, when the synchronized region is bounded, the value of $R$ first remains invariant ( $N>\sqrt{4 a \pi^{2} / d}$ ), and then increases with the increase of $N$ (when $N>\sqrt{4 a \pi^{2} / d}$ ), in which the synchronizability of the Networks-A remains constant at first and then weakens.

\section{Analysis of the Synchronizability of Networks-B}

\subsection{The Eigenvalue Spectrum and Synchronizability of Networks-B}

From the structural model in Figure 1(b), the supra-Laplacian matrix corresponding to Networks-B can be written as: 

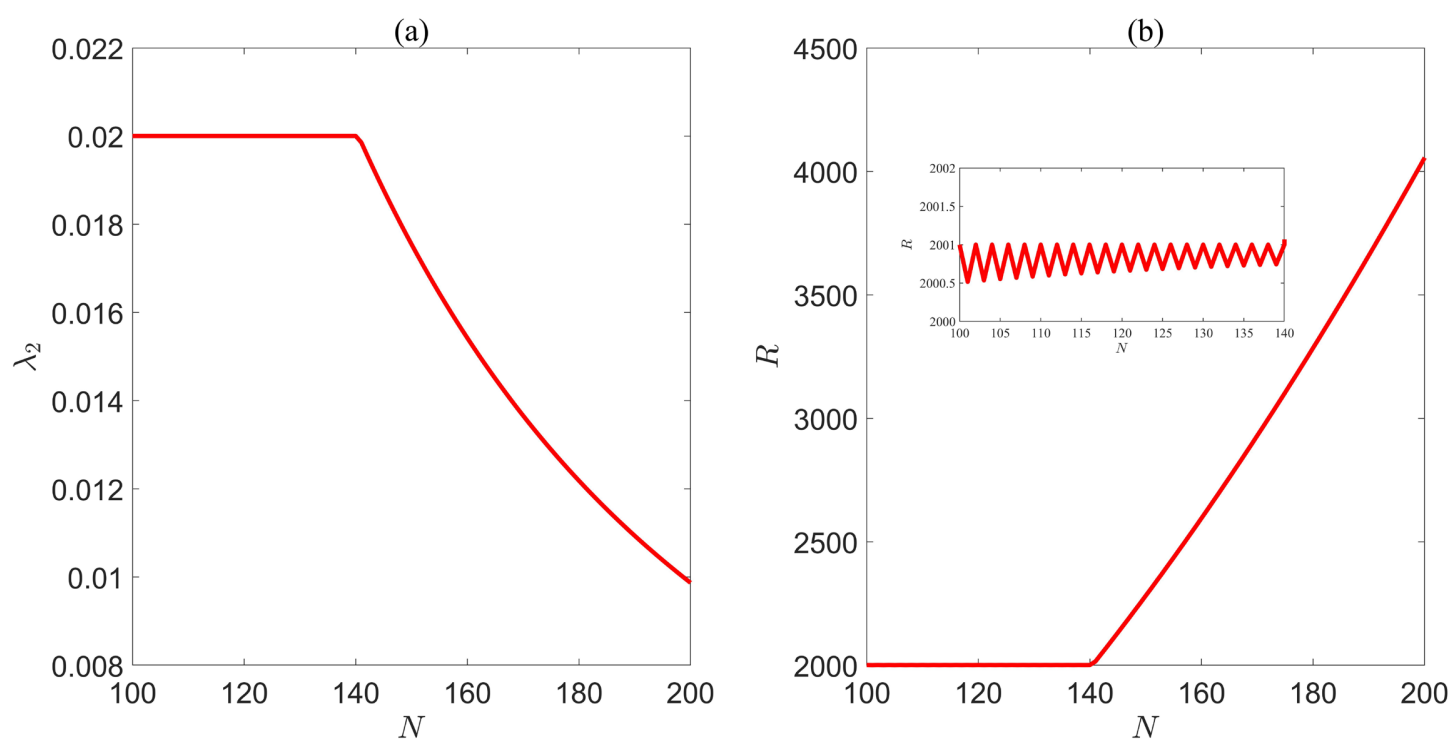

Figure 4. The synchronizability of Networks-A vs. varying the number of nodes within each layer $N$ ( $a=10, d=0.02)$. (a) $\lambda_{2}$ vs. varying $N(N$ varies from 100 to 200$)$; (b) $R$ vs. varying $N$ ( $N$ varies from 100 to 200) (subgraph: $R$ vs. varying $N$ ( $N$ varies from 100 to 140$)$ ).

$$
\mathcal{L}_{2}=\left(\begin{array}{cc}
A_{1}+d I_{N} & -d I_{N} \\
-d I_{N} & A_{1}^{\mathrm{T}}+d I_{N}
\end{array}\right)
$$

where

$$
A_{1}=\left(\begin{array}{ccccc}
a & -a & 0 & \cdots & 0 \\
0 & a & -a & \cdots & 0 \\
\vdots & \vdots & \ddots & \ddots & \vdots \\
0 & \cdots & 0 & a & -a \\
-a & 0 & \cdots & 0 & a
\end{array}\right) .
$$

According to Lemma 2, we can get the Characteristic polynomials of $\mathcal{L}_{2}$ is:

$$
\begin{aligned}
& \left|\lambda I_{2 N}-\mathcal{L}_{2}\right| \\
& =\left|\begin{array}{cc}
\lambda I_{N}-\left(A_{1}+d I_{N}\right) & d I_{N} \\
d I_{N} & \lambda I_{N}-\left(A_{1}^{\mathrm{T}}+d I_{N}\right)
\end{array}\right| \\
& =\left|\left(\lambda I_{N}-d I_{N}-A_{1}\right) \cdot\left(\lambda I_{N}-d I_{N}-A_{1}^{\mathrm{T}}\right)-d^{2} I_{N}\right| \\
& =\left|\begin{array}{cccc}
(\lambda-a-d)^{2}+a^{2}-d^{2} & a(\lambda-a-d) & \overbrace{0 \cdots 0}^{N-3} & a(\lambda-a-d) \\
a(\lambda-a-d) & (\lambda-a-d)^{2}+a^{2}-d^{2} & a(\lambda-a-d) & \overbrace{}^{N-3} \\
\ddots & \ddots & \ddots & \ddots \\
a(\lambda-a-d) & \overbrace{0 \cdots 0}^{N-3} & a(\lambda-a-d) & (\lambda-a-d)^{2}+a^{2}-d^{2}
\end{array}\right|
\end{aligned}
$$

Let $\left|\lambda I_{2 N}-\mathcal{L}_{2}\right|=0$, the eigenvalues of $\mathcal{L}_{2}$ can be written as [32]:

$$
\lambda_{l}=d+2 a \sin ^{2}\left(\frac{(l-1) \pi}{N}\right) \pm \sqrt{d^{2}-a^{2} \sin ^{2}\left(\frac{2(l-1) \pi}{N}\right)}, \quad l=1,2, \cdots, N .
$$


To make $d^{2}-a^{2} \sin ^{2} \frac{2(l-1) \pi}{N} \geq 0$, just consider the case $d \geq a$. The minimum non-zero eigenvalue $\lambda_{2}$ of $\mathcal{L}_{2}$ is at $l=2$ :

$$
\lambda_{2}=d+2 a \sin ^{2} \frac{\pi}{N}-\sqrt{d^{2}-a^{2} \sin ^{2} \frac{2 \pi}{N}} .
$$

when $N$ is odd, at $l=\frac{N-1}{2}+1$ or $l=\frac{N+1}{2}+1$ has a maximum value of $\lambda$ :

$$
\lambda_{\max }=d+2 a \cos ^{2} \frac{\pi}{2 N}+\sqrt{d^{2}-a^{2} \sin ^{2} \frac{\pi}{N}} .
$$

when $N$ is even, at $l=\frac{N}{2}+1$ has a maximum value of $\lambda$ :

$$
\lambda_{\max }=2(a+d) .
$$

In actuality, the scale of networks is huge and for simplicity we take:

$$
R=\frac{\lambda_{\max }}{\lambda_{2}} \approx \frac{\lambda_{\max } \approx 2(a+d) .}{d+2 a \sin ^{2} \frac{\pi}{N}-\sqrt{d^{2}-a^{2} \sin ^{2} \frac{2 \pi}{N}}} .
$$

According to the MSF theory, the relationship between the synchronizability of Networks-B and the structural parameters is shown in Table 2.

In $a=d$, the relationship between the synchronizability of Networks-B and the structural parameters can be transformed from Table 2 to Table 3.

\subsection{Numerical Simulation of the Synchronizability of Networks-B}

When $a=d$, with the unbounded synchronized region, Figure 5(a) shows that the synchronizability of Networks- $B$ is strengthened because $\lambda_{2}$ becomes larger with increases in $a, d$. Figure 6(a) shows that the synchronizability of Networks-B is weakened because $\lambda_{2}$ becomes smaller with increases $N$. When the

\begin{tabular}{|c|c|c|c|c|}
\hline & & \multicolumn{3}{|c|}{ Increase of } \\
\hline & & $a$ & $d$ & $N$ \\
\hline $\begin{array}{c}\text { The case of the } \\
\text { synchronized region } \\
\text { unbounded }\end{array}$ & $\lambda_{2}=d+2 a \sin ^{2} \frac{\pi}{N}-\sqrt{d^{2}-a^{2} \sin ^{2} \frac{2 \pi}{N}}$ & $\uparrow$ & $\downarrow$ & $\downarrow$ \\
\hline The case of the & $2(a+d)$ & & & \\
\hline $\begin{array}{l}\text { synchronized region } \\
\text { bounded }\end{array}$ & $\lambda_{2} d+2 a \sin ^{2} \frac{\pi}{N}-\sqrt{d^{2}-a^{2} \sin ^{2} \frac{2 \pi}{N}}$ & $\uparrow$ & $\downarrow$ & $\downarrow$ \\
\hline
\end{tabular}
bounded synchronized region, as is shown in Figure 5(b) and Figure 6(b), the synchronizability of Networks-B is only determined by the number of nodes within

Table 2. The change of synchronizability of Networks-B with the increase of $a, d, N$ when $d \geq a$.

—: unchanged; $\uparrow:$ strengthen; $\downarrow$ : weaken. 
Table 3. The change of synchronizability of Networks-B with the increase of $a, d, N$ when $a=d$.

\begin{tabular}{|c|c|c|c|}
\hline & & \multicolumn{2}{|c|}{ Increase of } \\
\hline & & $a, d$ & $N$ \\
\hline The case of the synchronized region unbounded & $\lambda_{2} \approx \frac{4 a \pi^{2}}{N^{2}}$ & $\uparrow$ & $\downarrow$ \\
\hline The case of the synchronized region bounded & $R \approx \frac{N^{2}}{\pi^{2}}$ & - & $\downarrow$ \\
\hline
\end{tabular}

—: unchanged; $\uparrow:$ strengthen; $\downarrow$ : weaken.
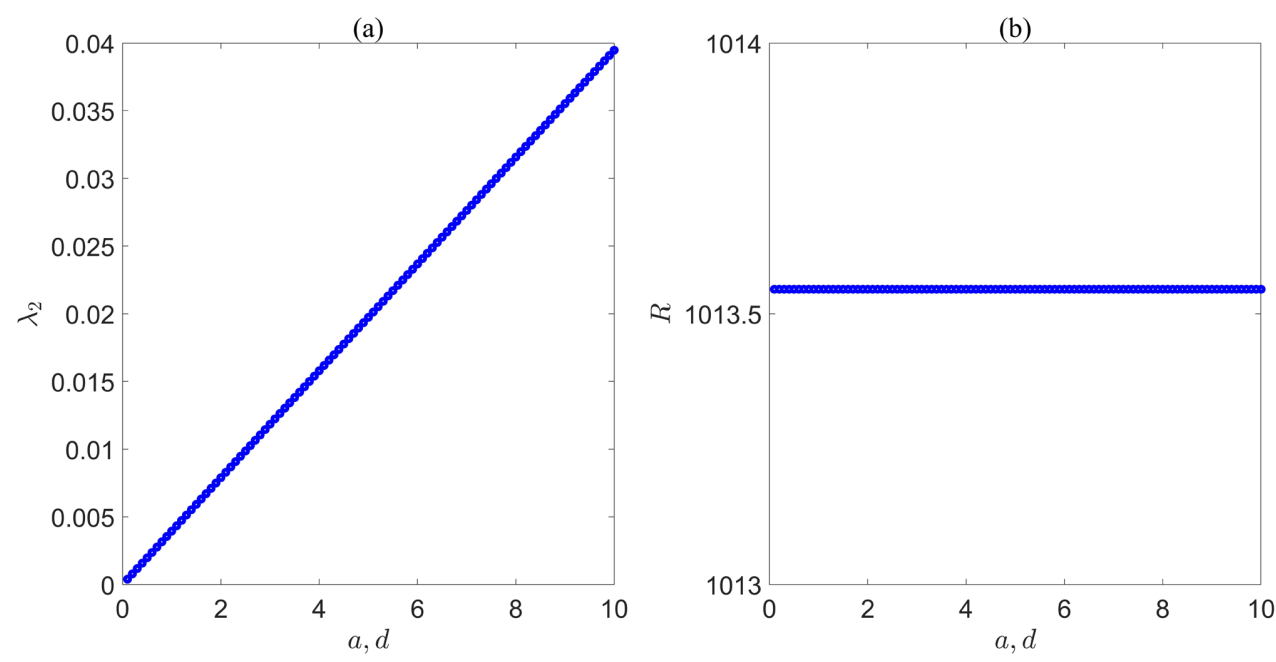

Figure 5. The synchronizability of Networks-B vs. varying coupling weight $a, d$ when $a=d$ ( $N=100$ ). (a) $\lambda_{2}$ vs. varying $a, d$ ( $a, d$ varies from 0.01 to 10 ); (b) $R$ vs. varying $a, d$ ( $a, d$ varies from 0.01 to 10$)$.
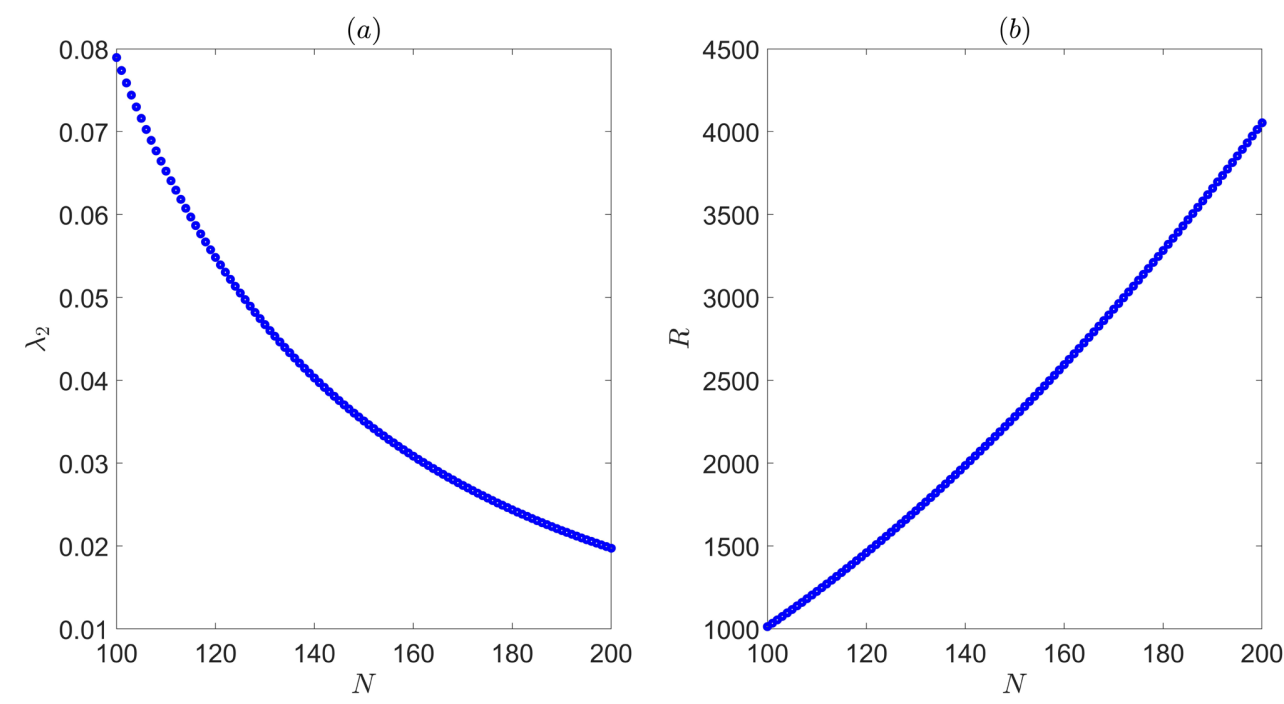

Figure 6. The synchronizability of Networks-B vs. varying the number of nodes within each layer $N$ when $a=d \quad(a=d=20)$. (a) $\lambda_{2}$ vs. varying $N$ ( $N$ varies from 100 to 200); (b) $R$ vs. varying $N$ ( $N$ varies from 100 to 200$)$. 
each layer $N$. The value $R$ increases with the increase of $N$, the synchronizability of Networks-B is weakened. When $N=100$ the value $R \approx N^{2} / \pi^{2} \approx 1013.212$ is shown in Figure 5(b).

When $d>a$ it is observed that the value $\lambda_{2}$ increases with the increase of $a$ (Figure 7(a)) and the value $R$ decreases exponentially with the increase of $a$ (Figure $7(b)$ ). This means that, with the bounded or unbounded synchronized region, the synchronizability of Networks-B is enhanced continuously with an increase in $a$. Figure 8 and Figure 9 show the change of synchronizability of
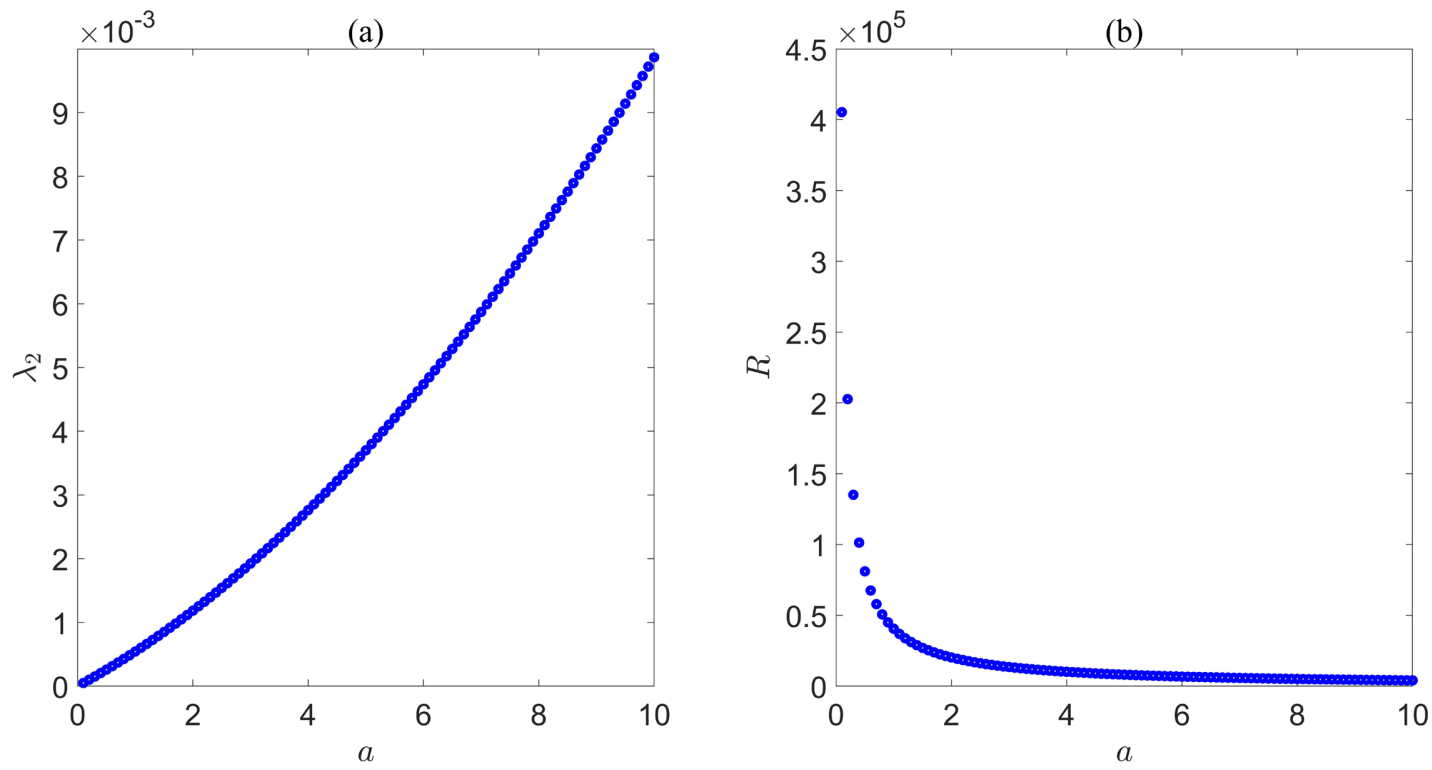

Figure 7. The synchronizability of Networks-B vs. varying intra-layer coupling weight $a \quad(N=200, d=10)$. (a) $\lambda_{2}$ vs. varying a ( $a$ varies from 0.01 to 10 ); (b) $R$ vs. varying $a$ ( $a$ varies from 0.01 to 10).
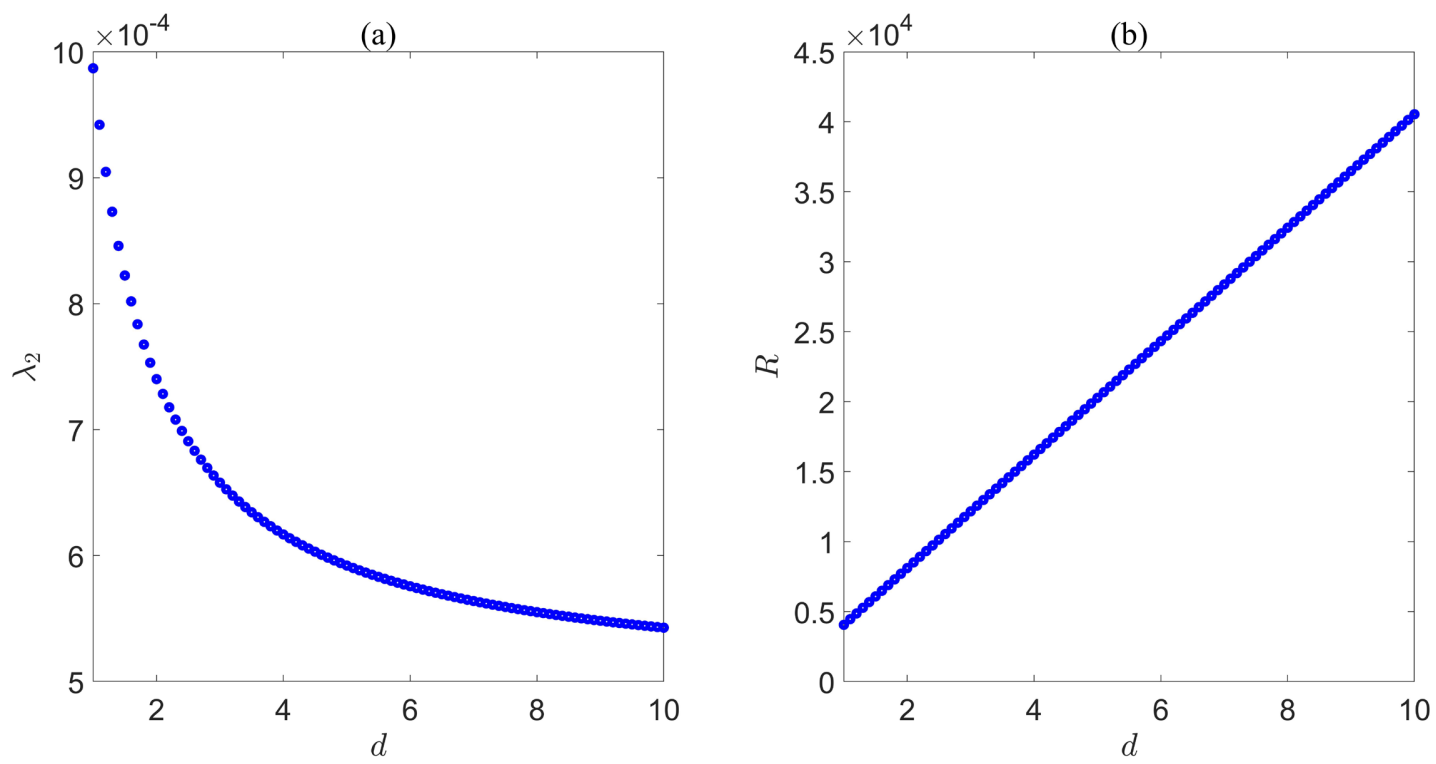

Figure 8. The synchronizability of Networks-B vs. varying inter-layer coupling weight $d(N=200, a=1)$. (a) $\lambda_{2}$ vs. varying $d$ ( $d$ varies from 1 to 10 ); (b) $R$ vs. varying $d$ ( $d$ varies from 1 to 10 ). 

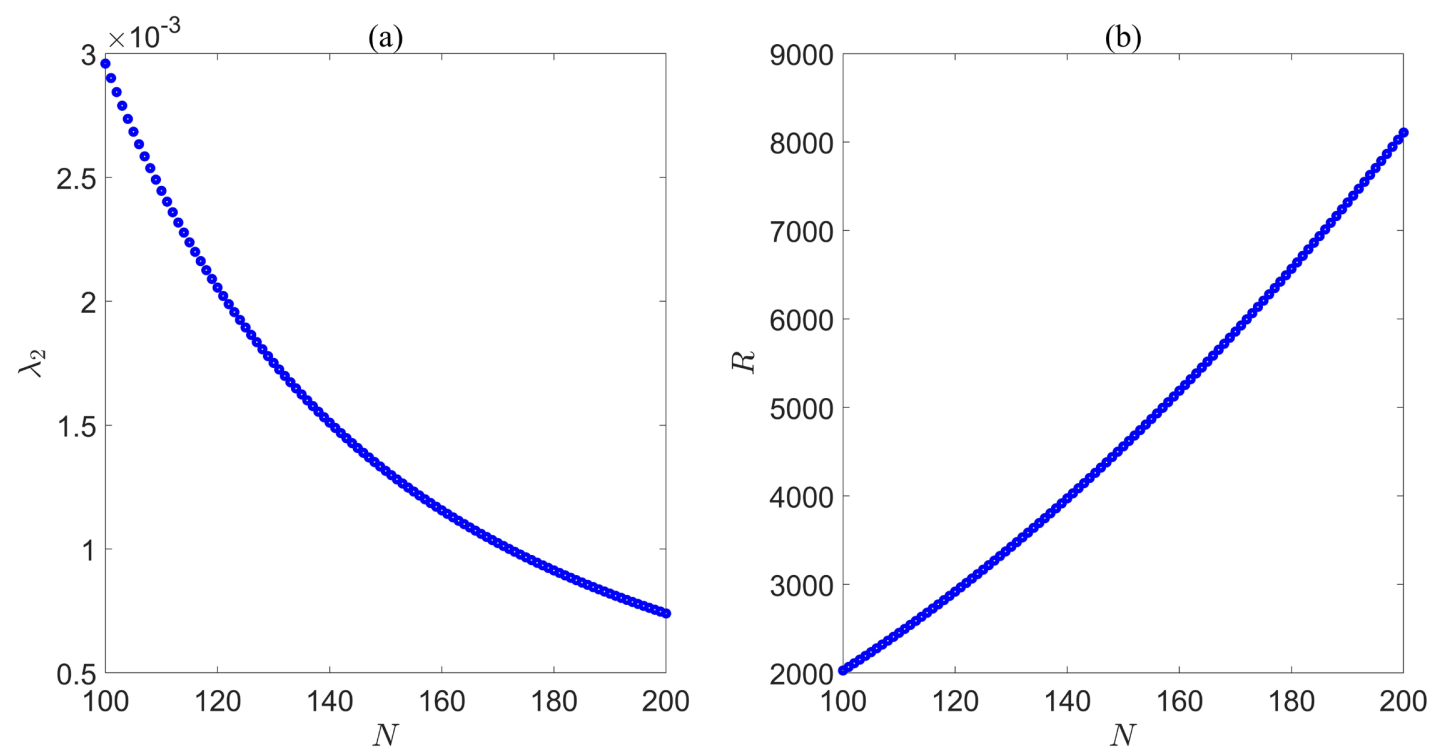

Figure 9. The synchronizability of Networks-B vs. varying the number of nodes within each layer $N$ ( $a=1, d=2$ ). (a) $\lambda_{2}$ vs. varying $N$ ( $N$ varies from 100 to 200$)$; (b) $R$ vs. varying $N$ ( $N$ varies from 100 to 200$)$.

Networks-B with the increase of $d$ and $N$. The value $\lambda_{2}$ decreases with the increase of $d$ and $N$ (as shown in Figure 8(a) and Figure 9(a)). The value of $R$ increases monotonically with the increase of $d$ and $N$ (as shown in Figure 8(b) and Figure 9(b)). Regardless of whether the synchronized region is bounded or unbounded, the synchronizability of Networks-B is weakened with the increase in $d$ and $N$.

The combined Figures 7-9, it can be noticed that the intra-layer coupling weight $a$ has a greater effect on the synchronizability of Networks-B than the inter-layer coupling weight $d$ has on the synchronizability of Networks-B, so although the inter-layer coupling weight $d$ has a suppressive effect on the improvement of the synchronizability of Networks-B, the synchronizability of Networks-B is enhanced when $a, d$ increases simultaneously.

\section{The Comparison of Synchronizability of Four Kinds of Double-Layer Ring Networks}

In this section, we analyze the changes of synchronizability of four kinds of double-layer ring networks, namely, Networks-A, Networks-B, Networks-C, and Networks- $\mathrm{D}$, under parameters (the number of nodes within each layer $N$, the inter-layer coupling weight $d$, and the intra-layer coupling weight $a$ ) changes with the help of numerical calculation methods and simulation experiments because the eigenvalues of the supra-Laplacian matrix of Networks-D cannot be expressed analytically. Where the numerical simulation experiments of Networks-D use the interlayer random-added edge probability $p=1$. The value of $\lambda_{2}$ and $R$ of Networks-D in the tests are the results of 50 independent repetitions to take the average value. In the figure below, the Green triangular solid line (Networks-A) illustrates the double-layer inter-layer directed ring network; 
the black star solid line (Networks-B) illustrates the double-layer intra-layer directed ring network; the red circular dashed line (Networks-C) illustrates the double-layer undirected ring network; the blue quadrilateral solid line (Networks-D, $p=1$ ) illustrates the double-layer undirected ring network with inter-layer random-added-edge probability $p=1$.

For comparison purposes, the relationship between the synchronizability of the Networks-C and the structural parameters is shown in following Table 4 [27].

As illustrated in Figure 10, it is intuitive to show that the value $\lambda_{2}$ increases rapidly (Figure 10(a)) the value $R$ decreases swiftly (Figure 10(b)) with the increase of the inter-layer random-added-edge probability $p$. So, the synchronizability of Networks-D is continuously strengthened, whether the synchronized region is bounded or unbounded. Naturally, it can be observed that the larger the interlayer random edge addition probability $p$, the faster the synchronizability of Networks-D optimization.

As is shown in Figure 11(a), when $d>a$, with the unbounded synchronized region, for all four kinds of double-layer ring networks, the value $\lambda_{2}$ increases with the increase of $a$, so the synchronizability is strengthened with the increase of $a$. Then the size of the synchronizability of these four kinds of double-layer ring networks is: $\operatorname{Syn}_{D}>\operatorname{Syn}_{A}=\operatorname{Syn}_{C}>\operatorname{Syn}_{B}$. With the bounded synchronized region, it is observed from Figure 11(b) that the value $R$ decreases with the increase of $a$ all four kinds of double-layer ring networks. Then the size of the synchronizability of these four kinds of double-layer ring networks is:

$\operatorname{Syn}_{D}>\operatorname{Syn}_{A}>\operatorname{Syn}_{C}>\operatorname{Syn}_{B}$. In sum, whether the synchronized region is bounded or unbounded, the synchronizability of the four kinds of double-layer ring networks is enhanced continuously with the increase of intra-layer coupling weight $a$.
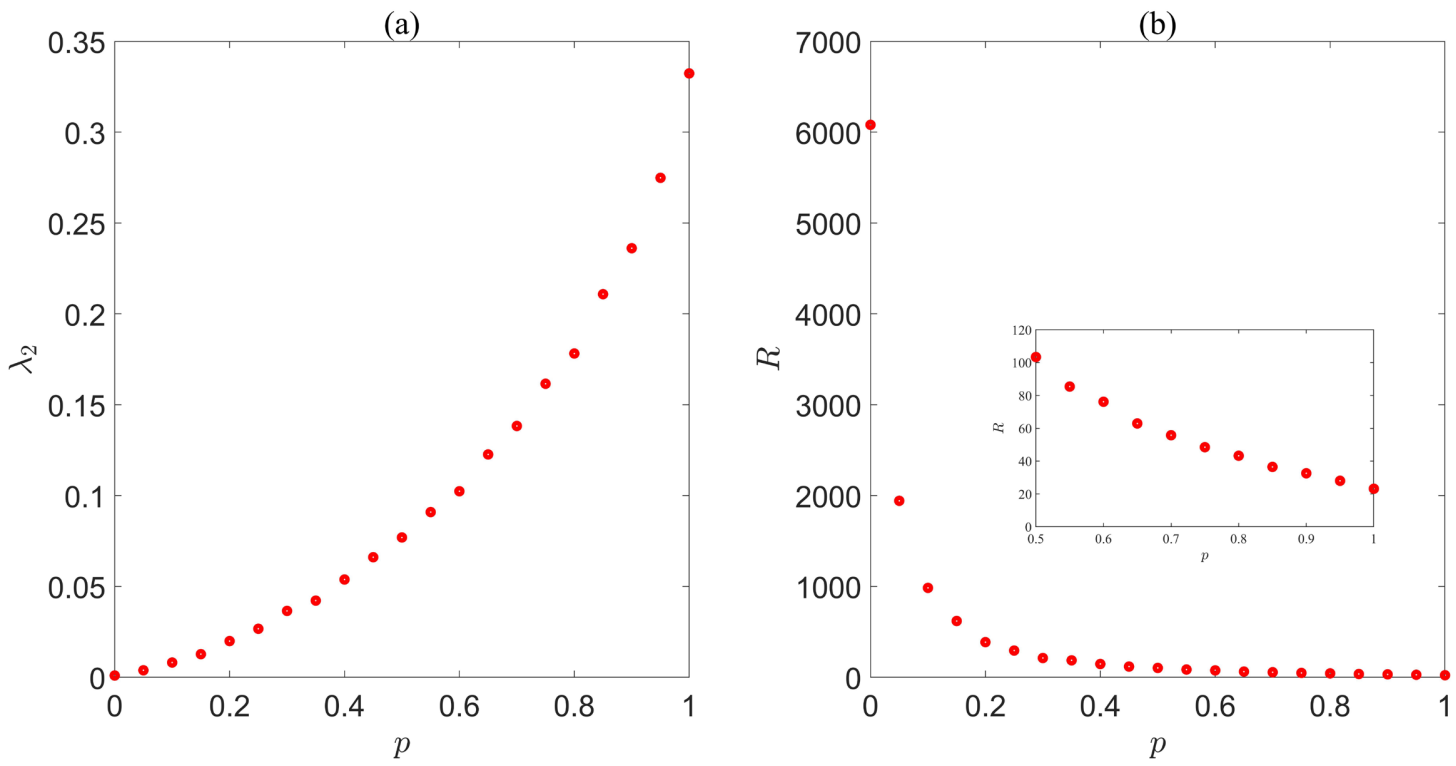

Figure 10. The synchronizability of Networks-D vs. varying inter-layer random-added-edge probability $p$ ( $N=200, a=1, d=1$ ). (a) $\lambda_{2}$ vs. varying $p$ ( $p$ varies from 0 to 1 ); (b) $R$ vs. varying $p$ ( $p$ varies from 0 to 1 ) (subgraph: $R$ vs. varying $p$ ( $p$ varies from 0.5 to 1$)$ ). 
Table 4. The change of synchronizability of Networks-C with the increase of $a, d, N$.

\begin{tabular}{|c|c|c|c|c|c|}
\hline & & & \multicolumn{3}{|c|}{ Increase of } \\
\hline & & & $a$ & $d$ & $N$ \\
\hline \multirow{2}{*}{$\begin{array}{c}\text { The case of } \\
\text { synchronized region } \\
\text { unbounded }\end{array}$} & $d<\frac{2 a \pi^{2}}{N^{2}}$ & $\lambda_{2} \approx 2 d$ & - & $\uparrow$ & - \\
\hline & $d>\frac{2 a \pi^{2}}{N^{2}}$ & $\lambda_{2} \approx \frac{4 a \pi^{2}}{N^{2}}$ & $\uparrow$ & - & $\downarrow$ \\
\hline \multirow{2}{*}{$\begin{array}{c}\text { The case of the } \\
\text { synchronized region } \\
\text { bounded }\end{array}$} & $d<\frac{2 a \pi^{2}}{N^{2}}$ & $R \approx \frac{a(3 \pi+2)+2 \pi d}{2 \pi d}$ & $\downarrow$ & $\downarrow$ & - \\
\hline & $d>\frac{2 a \pi^{2}}{N^{2}}$ & $R \approx \frac{a N^{2}(3 \pi+2)+2 \pi d N^{2}}{4 a \pi^{3}}$ & $\downarrow$ & $\uparrow$ & $\uparrow$ \\
\hline
\end{tabular}

—: unchanged; $\uparrow:$ strengthen; $\downarrow$ : weaken.

(a)

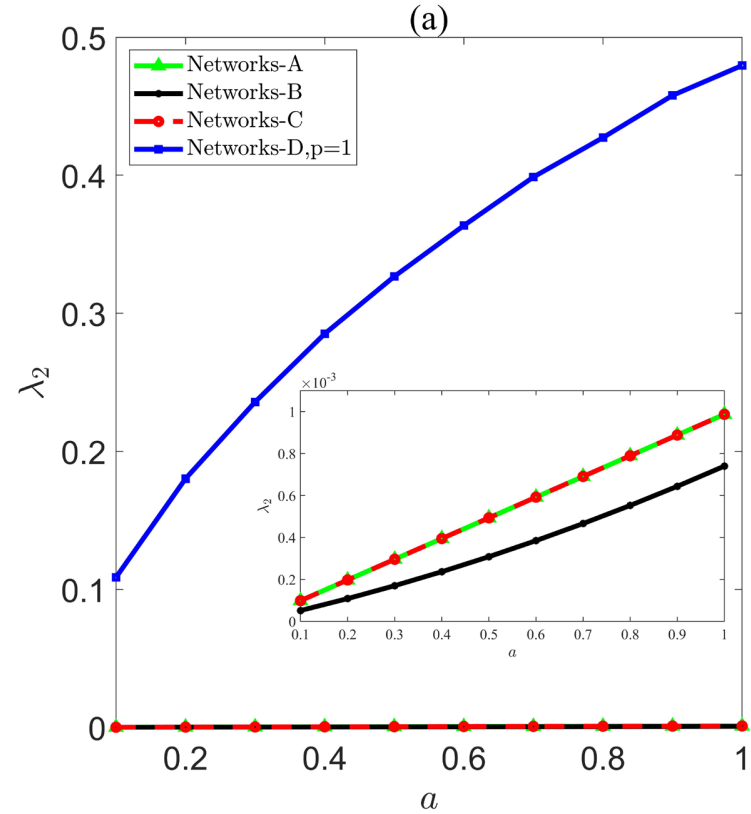

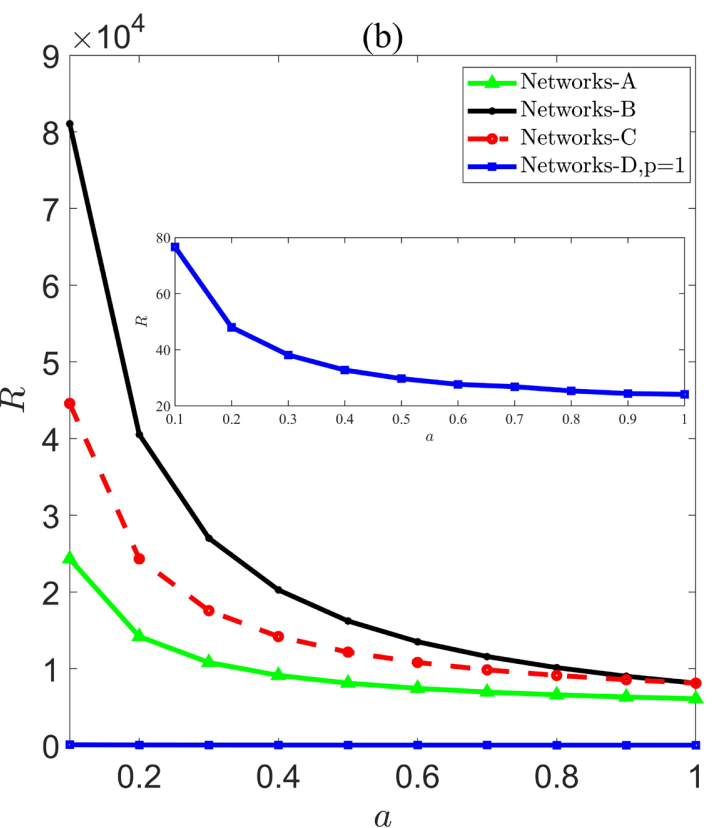

Figure 11. The synchronizability of four kinds of double-layer ring networks vs. varying intra-layer coupling weight $a$ $\left(N=200, d=2\right.$ ). (a) $\lambda_{2}$ vs. varying $a$ ( $a$ varies from 0.1 to 1$)$ (subgraph: $\lambda_{2}$ vs. varying $a$ ( $a$ varies from 0.1 to 1$)$ ); (b) $R$ vs. varying $a$ ( $a$ varies from 0.1 to 1 ) (subgraph: $R$ vs. varying $a$ ( $a$ varies from 0.1 to 1 )).

From Figure 12(a), it is shown that the $\lambda_{2}$ values of Networks-A and Networks-C do not change with an increase of $d$, the $\lambda_{2}$ value of Networks-B decreases slowly with the increase of $d$, and the $\lambda_{2}$ value of Networks-D increases with the increase of $d$ in the presence of inter-layer randomly added probability $p=1$. So, with the synchronized region is unbounded, the synchronizability of Networks-A and Networks- $\mathrm{C}$ is the same as the trend with the increase of $d$, the synchronizability of Networks-B is weakened with the increase of $d$, and the synchronizability of Networks-D is strengthened with the increase of $d$. The synchronizability of networks in descending order of magnitude is: 
(a)

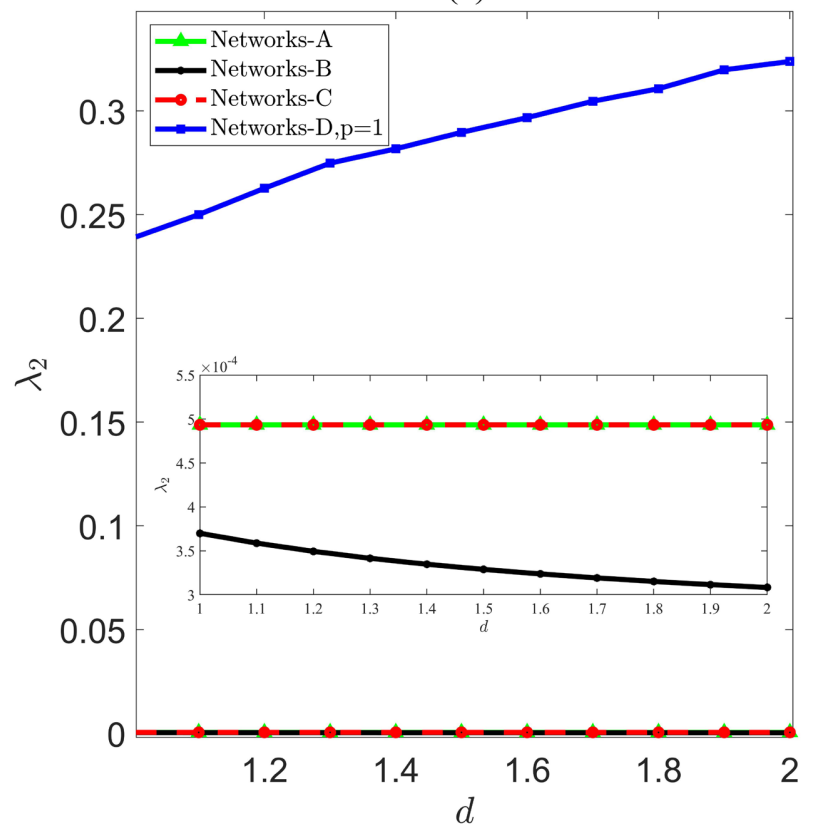

(b)

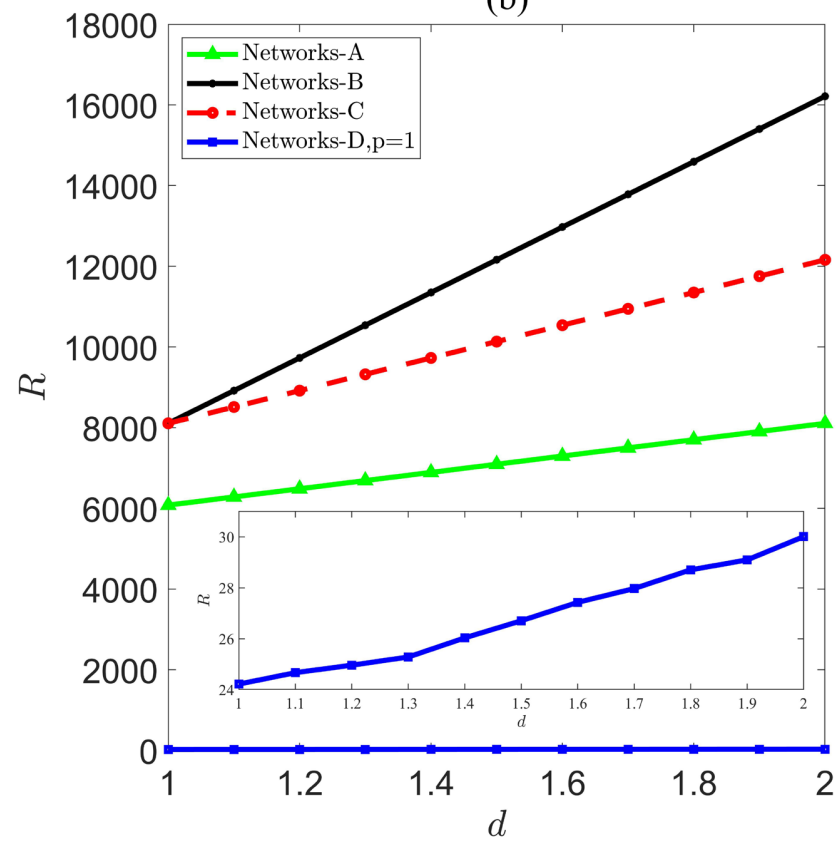

Figure 12. The synchronizability of four kinds of double-layer ring networks vs. varying inter-layer coupling weight $d$ ( $N=200, a=0.5$ ). (a) $\lambda_{2}$ vs. varying $d$ ( $d$ varies from 1 to 2 ) (subgraph: $\lambda_{2}$ vs. varying $d$ ( $d$ varies from 1 to 2 )); (b) $R$ vs. varying $d$ ( $d$ varies from 1 to 2 ) (subgraph: $R$ vs. varying $d$ ( $d$ varies from 1 to 2$)$ ).

$\operatorname{Syn}_{D}>\operatorname{Syn}_{A}=\operatorname{Syn}_{C}>\operatorname{Syn}_{B}$. In Figure 12(b), it is clear that the $R$ value of all four kinds of networks increases with the increase of $d$. Hence, the synchronizability of the four kinds of networks is weakened with the increase of $d$ (bounded synchronized region). So, it is obtained from Figure 12(b) that the size of synchronizability of these four kinds of double-layer ring networks is weakened with inter-layer coupling weight $d$ in the following order: $\operatorname{Syn}_{D}>S y n_{A}>S y n_{C}>S y n_{B}$.

When $d>a$ the synchronized region is unbounded, it can be seen from Figure 13(a) that the $\lambda_{2}$ values of these four kinds of double-layer ring networks all have a slowly decreasing trend with the increase of $N$. Then the $\lambda_{2}$ value $\left(\lambda_{2}=4 a \pi^{2} / N^{2}\right)$ of Networks-A and Networks-C have the same trend with the increase of $N$. So, the synchronizability of the four kinds of double-layer ring networks is diminished with the increase $N$. In particular, it is found that the order of these four kinds of double-layer ring network synchronizability from largest to smallest is the same as in Figure 11(a) and Figure 12(a). With the synchronized region is bounded, from Figure 13(b), it is clear that $R_{B}=R_{C}>R_{A}>R_{D}$ for any the number of nodes within each layer $N$. Then $R$ value ( $R \approx 2 N^{2} / \pi^{2}$ ) of Networks-B and Networks-C have the same trend with the increase of $N$.

In contrast to Figure 13, Figure 14 shows the change of the synchronizability of these four kinds of double-layer ring networks with the increase of the number of nodes within each layer $N$ for the case $a=d$. With the synchronized region is unbounded, from Figure 14(a), the $\lambda_{2}$ values $\left(\lambda_{2}=4 a \pi^{2} / N^{2}\right)$ of Networks-A, Networks-B, and Networks-C have the same trend with the increase of 

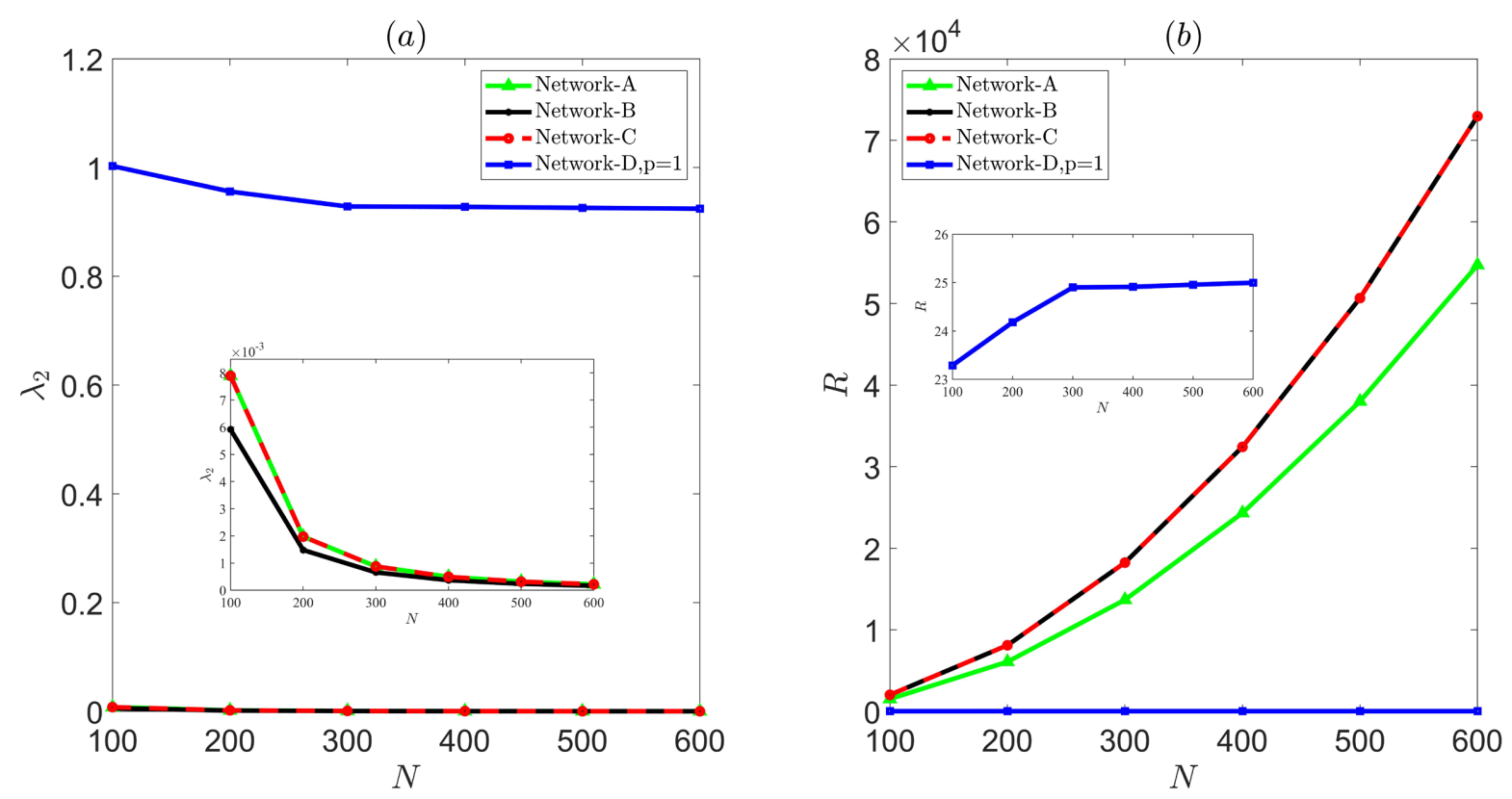

Figure 13. The synchronizability of four kinds of double-layer ring networks vs. varying the number of nodes within each layer $N(a=2, d=4)$. (a) $\lambda_{2}$ vs. varying $N$ ( $N$ varies from 100 to 600) (subgraph: $R$ vs. varying $N(N$ varies from 100 to 600$)$ ); (b) $R$ vs. varying $N(N$ varies from 100 to 600) (subgraph: $R$ vs. varying $N(N$ varies from 100 to 600$)$ ).
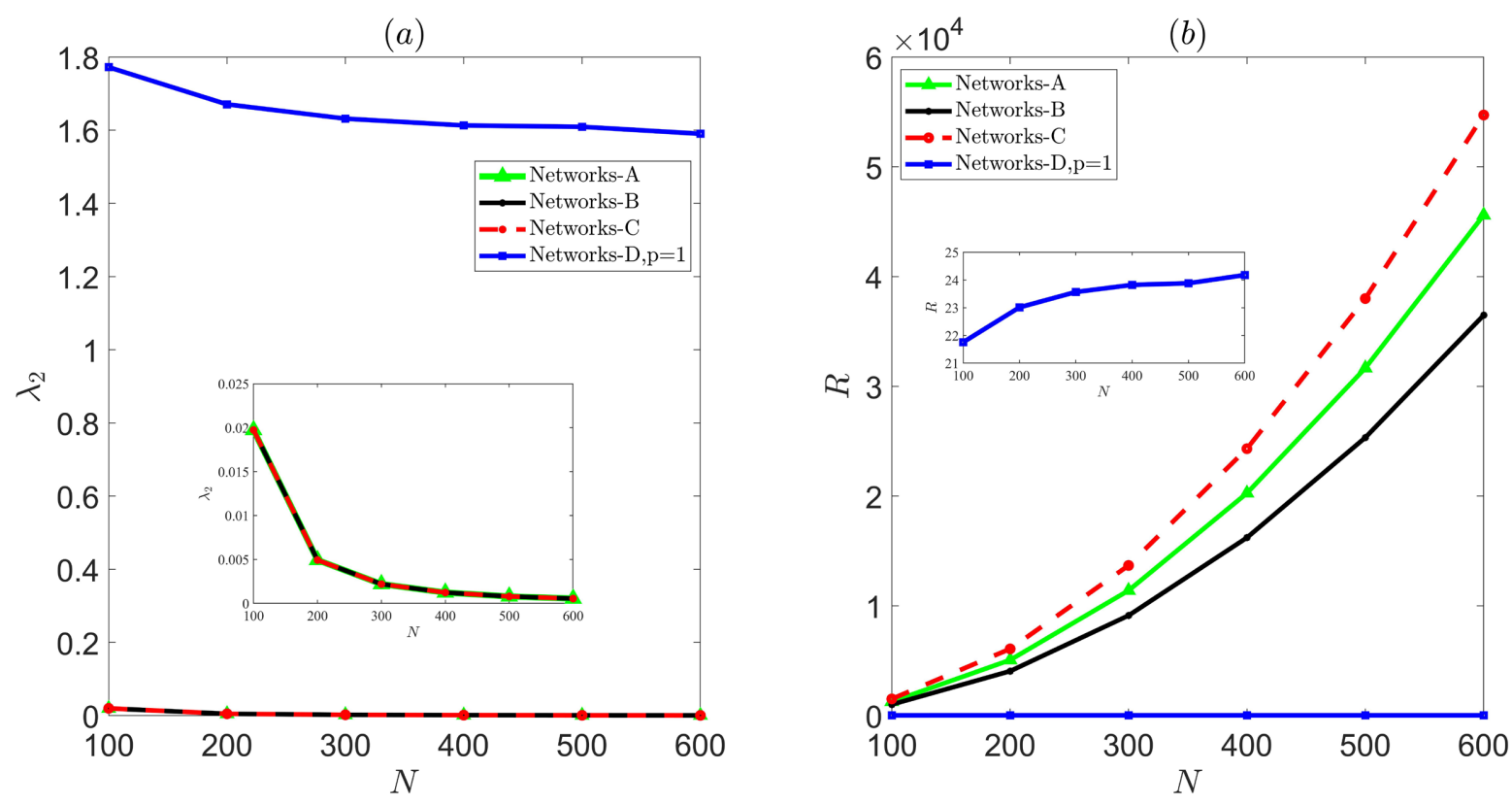

Figure 14. The synchronizability of four kinds of double-layer ring networks vs. varying the number of nodes within each layer $N$ when $a=d \quad(a=d=5)$. (a) $\lambda_{2}$ vs. varying $N(N$ varies from 100 to 600) (subgraph: $R$ vs. varying $N(N$ varies from 100 to 600$)$ ); (b) $R$ vs. varying $N$ ( $N$ varies from 100 to 600 ) (subgraph: $R$ vs. varying $N$ ( $N$ varies from 100 to 600$)$ ).

$N$. The $\lambda_{2}$ value of Networks-D decreases slightly in the presence of inter-layer randomly added probabilities $p=1$ with the increase of $N$. The size of the synchronizability of these four kinds of networks is, in order,

$\operatorname{Syn}_{D}>\operatorname{Syn}_{A}=\operatorname{Syn}_{C}>\operatorname{Syn}_{B}$. With the synchronized region is unbounded, the 
value $R$ increases with the increase of $N$ as shown in Figure 14(b), so the synchronizability of all four kinds of networks is monotonically weakened.

Combining Figure 13 and Figure 14, the four kinds of double-layer ring networks have a different ranking of synchronizability with $N$ for different $a$ and $d$. However, in general, the synchronizability of all four kinds of networks is in the following order: $\operatorname{Syn}_{D}>S y n_{A}=S y n_{C}>S y n_{B}$, with the synchronized region is unbounded. It is easy to conclude that the ranks for synchronizability are: $\operatorname{Syn}_{D}>\operatorname{Syn}_{B}>\operatorname{Syn}_{A}>\operatorname{Syn}_{C}$, with the synchronized region is bounded.

\section{Conclusions}

For Networks-A and Networks-B, firstly, we investigate the synchronizability of inter-layer directed double-layer ring networks (Networks-A) and intra-layer directed double-layer ring networks (Networks-B), and rigorously derive the effects of each parameter on the synchronizability of the two kinds of networks, giving the analytical expressions for the eigenvalues. Finally, the theory is verified by numerical simulation analysis. The results show that the $R$ values of the ring network are affected by both odd and even numbers in a small network range, which is similar to the results of the analytical expression for the eigenvalues of the network derived in [28]. By varying a single parameter, an optimal value of the parameter was found, which led to the optimal synchronizability of the networks. The effects of changes in inter-layer coupling weight $a$, intra-layer coupling weight $d$, the number of nodes within each layer $N$ upon the network synchronizability for Networks-A and Networks-B are similar. To sum up, the larger the intra-layer coupling weight, the smaller the inter-layer coupling weight, and the smaller the number of nodes is more preferable to the synchronization of Network-A and Networks-B.

Then, we also find that Networks-A, Networks- $B$, and Networks- $C$ have the same minimum non-zero eigenvalue $\lambda_{2}$ when the inter-layer coupling weight $d$ and intra-layer coupling weight $a$ are equal, indicating that these three kinds of networks have the same synchronizability when the synchronized region is unbounded. In addition, the synchronizability of Networks-D increases as the probability of randomly adding edges between layers increases. More importantly, we compare the synchronizability of four kinds of double-layer ring networks and find that the synchronizability of Networks- $\mathrm{D}$ is the best for each parameter variation, while it is remaining three kinds of ring networks are comparable, and the synchronizability of Networks-A is stronger compared to Networks-B and Networks-C when both intra-layer coupling weight $a$ and inter-layer coupling weight $d$ are affected. Recently, the diffusion of networks is an interesting and challenging topic. The spectrum of eigenvalues of multilayer directed networks is in general complex; it is part of our future work to investigate the diffusion dynamics of multiplex directed networks.

\section{Acknowledgements}

This project is supported by the National Natural Science Foundation of China 
(No. 61563013) and the Natural Science Foundation of Guangxi (No. 2018GXNSFAA138095).

\section{Conflicts of Interest}

The authors declare no conflicts of interest regarding the publication of this paper.

\section{References}

[1] De Domenico, M., Sole-Ribalta, A., Omodei, E., et al. (2015) Ranking in Interconnected Multilayer Networks Reveals Versatile Nodes. Nature Communications, 6, Article No. 6868. https://doi.org/10.1038/ncomms7868

[2] Granell, C., Gomez, S. and Arenas, A. (2013) Dynamical Interplay between Awareness and Epidemic Spreading in Multiplex Networks. Physical Review Letters, 111, Article ID: 128701. https://doi.org/10.1103/PhysRevLett.111.128701

[3] Buldyrev, S.V., Parshani, R., Paul, G., et al. (2010) Catastrophic Cascade of Failures in Interdependent Networks. Nature, 464, 1025-1028.

https://doi.org/10.1038/nature08932

[4] Zhang, X.Q., Zhang, Z.K., Wang, W.B., et al. (2021) Multiplex Network Reconstruction for the Coupled Spatial Diffusion of Infodemic and Pandemic of COVID-19. International Journal of Digital Earth, 14, 401-423. https://doi.org/10.1080/17538947.2021.1888326

[5] Feng, P.H., Gong, Z.W. and Sun, D.Y. (2019) Research on Interpersonal Relationship Network of Pyramid Scheme Organization Based on Social Network: A Case Study. International Journal of Modern Physics C, 30, Article ID: 1940016. https://doi.org/10.1142/S0129183119400163

[6] Yu, W.W., Cao, J.D., Chen, G.R., et al. (2009) Local Synchronization of a Complex Network Model. IEEE Transactions on Systems, Man, and Cybernetics Part B (Cybernetics), 39, 230-241. https://doi.org/10.1109/TSMCB.2008.2004964

[7] Kurant, M. and Thiran, P. (2006) Layered Complex Networks. Physical Review Letters, 96, Article ID: 138701. https://doi.org/10.1103/PhysRevLett.96.138701

[8] Wu, X.Q., Li, Y.N., Wei, J., et al. (2020) Inter-Layer Synchronization in Two-Layer Networks via Variable Substitution Control. Journal of the Franklin Institute, 357, 2371-2387. https://doi.org/10.1016/j.jfranklin.2019.12.019

[9] Leyva, I., Sevilla-Escoboza, R., Sendiña-Nadal, I., Gutiérrez, R., Buldú, J.M. and Boccaletti, S. (2017) Inter-Layer Synchronization in Non-Identical Multi-Layer Networks. Scientific Reports, 7, Article No. 45475. https://doi.org/10.1038/srep45475

[10] Tang, L.K., Wu, X.Q., Lü, J.H., et al. (2019) Master Stability Functions for Complete, Intralayer, and Interlayer Synchronization in Multiplex Networks of Coupled Rössler Oscillators. Physical Review E, 99, Article ID: 012304. https://doi.org/10.1103/PhysRevE.99.012304

[11] Yu, W.W., Lyu, J.H., Yu, X.H., et al. (2015) Distributed Adaptive Control for Synchronization in Directed Complex Networks. SIAM Journal on Control and Optimization, 53, 2980-3005. https://doi.org/10.1137/140970781

[12] Wang, Y.W., Bian, T., Xiao, J.W., et al. (2015) Global Synchronization of Complex Dynamical Networks through Digital Communication with Limited Data Rate. IEEE Transactions on Neural Networks and Learning Systems, 26, 2487-2499. https://doi.org/10.1109/TNNLS.2014.2387443 
[13] Gomez-Gardenes, J., Gomez, S., Arenas A., et al. (2011) Explosive Synchronization Transitions in Scale-Free Networks. Physical Review Letters, 106, Article ID: 128701. https://doi.org/10.1103/PhysRevLett.106.128701

[14] Fan, C.X., Jiang, G.P. and Jiang, F.H. (2010) Synchronization between Two Complex Dynamical Networks Using Scalar Signals under Pinning Control. IEEE Transactions on Circuits and Systems I: Regular Papers, 57, 2991-2998. https://doi.org/10.1109/TCSI.2010.2048774

[15] Li, J.Y., Luan, Y.Y., Wu, X.Q., et al. (2021) Synchronizability of Double-Layer Dumbbell Networks. Chaos, 31, Article ID: 073101. https://doi.org/10.1063/5.0049281

[16] Deng, Y., Jia, Z., Deng, G.M., et al. (2020) Eigenvalue Spectrum and Synchronizability of Multiplex Chain Networks. Physica A: Statistical Mechanics and Its Applications, 537, Article ID: 122631. https://doi.org/10.1016/j.physa.2019.122631

[17] Xu, Y.H., Wu, X.Q., Li, N., et al. (2020) Fixed-Time Synchronization of Complex Networks with a Simpler Nonchattering Controller. IEEE Transactions on Circuits and Systems II: Express Briefs, 67, 700-704. https://doi.org/10.1109/TCSII.2019.2920035

[18] Fan, H.W., Kong, L.W., Wang, X.G., et al. (2020) Synchronization within Synchronization: Transients and Intermittency in Ecological Networks. National Science Review, 8, Article ID: nwaa269. https://doi.org/10.1093/nsr/nwaa269

[19] Wang, L., Jia, X.Y., Pan, X.Y. and Xia, C.Y. (2020) Extension of Synchronizability Analysis Based on Vital Factors: Extending Validity to Multilayer Fully Coupled Networks. Chaos, Solitons \& Fractals, 142, Article ID: 110484.

[20] Deng, Y., Jia, Z. and Liao, L. (2019) Synchronizability of Two-Layer Cluster Ring Networks. Communications \& Network, 11, 35-51.

https://doi.org/10.4236/cn.2019.112004

[21] Hang, L., Zhen, J. and Long, Y. (2017) The Synchronizability and Optimized Analysis of the Hierarchical Network. Communications and Network, 9, 207-218. https://doi.org/10.4236/cn.2017.93015

[22] Arenas, A., Diaz-Guilera, A., Kurths, J., et al. (2008) Synchronization in Complex Networks. Physics Reports, 469, 93-153. https://doi.org/10.1016/j.physrep.2008.09.002

[23] Wang, X., Li, X., et al. (2012) Network Science: An Introduction. High Education Press, Beijing.

[24] Wei, J., Wu, X.Q., Lu, J.A. and Wei, X. (2017) Synchronizability of Duplex Regular Networks. Europhysics Letters, 120, Article ID: 20005.

https://doi.org/10.1209/0295-5075/120/20005

[25] Sun, J., Li, X.X., Zhang, J.H., et al. (2017) Synchronizability and Eigenvalues of Multilayer Star Networks through Unidirectionally Coupling. Acta Physica Sinica (Chinese Edition), 66, Article ID: 188901. https://doi.org/10.7498/aps.66.188901

[26] Yang, L.X., Jiang, J. and Liu, X.J. (2019) Impacts of Node Arrangements on Synchronization of a Ring Oscillatory Power Network. Chaos, Solitons \& Fractals, 126, 60-65. https://doi.org/10.1016/j.chaos.2019.06.003

[27] Zhang, L. and Wu, Y. (2020) Synchronizability of Multilayer Networks with K-Nearest-Neighbor Topologies. Frontiers in Physics, 8, Article ID: 571507. https://doi.org/10.3389/fphy.2020.571507

[28] Yang, F.M., Jia, Z. and Deng, Y. (2021) Eigenvalue Spectrum and Synchronizability of Two Types of Double-Layer Star-Ring Networks with Hybrid Directional Coupling. Discrete Dynamics in Nature and Society, 2021, Article ID: 6623648. 
https://doi.org/10.1155/2021/6623648

[29] Gomez, S., Diaz-Guilera, A., Gomez-Gardenes, J., et al. (2013) Diffusion Dynamics on Multiplex Networks. Physical Review Letters, 110, Article ID: 28701.

https://doi.org/10.1103/PhysRevLett.110.028701

[30] Liu, H., Lu, J.A., Lü, J., et al. (2009) Structure Identification of Uncertain General Complex Dynamical Networks with Time Delay. Automatica, 45, 1799-1807. https://doi.org/10.1016/j.automatica.2009.03.022

[31] Lightstone, A.H. (1966) Fundamentals of Linear Algebra. Appleton-Century-Crofts, New York.

[32] Tejedor, A., Longjas, A., Foufoula-Georgiou, E., et al. (2018) Diffusion Dynamics and Optimal Coupling in Multiplex Networks with Directed Layers. Physical Review X, 8, Article ID: 031071. https://doi.org/10.1103/PhysRevX.8.031071 